Rev. Mus. Argentino Cienc. Nat., n.s.

2(1): $1-15,2000$

Buenos Aires, ISSN 1514-5158

\title{
Biogeographic delimitation of the Subantarctic subregion and its provinces
}

\author{
Juan J. MORRONE
}

Museo de Zoología, Facultad de Ciencias, UNAM, Apdo. Postal 70-399, 04510 México D.F., México.

\begin{abstract}
The Subantarctic subregion, which belongs to the Andean region of the Austral kingdom, comprises the austral Andes from $37^{\circ}$ south latitude to Cabo de Hornos, the archipelago of southern Chile and Argentina, and the Malvinas, South Georgia, and Juan Fernández islands. The Subantarctic subregion comprises six provinces: Maule (southern Chile and Argentina, between 34-37 south latitude), Valdivian (southern Chile and Argentina, south of the Maule province, reaching $47^{\circ}$ south latitude), Magellanic forest (southem Chile from $47^{\circ}$ south latitude to Cabo de Hornos, and sotthern Argentina in small portions of western Santa Cruz and Tierra del Fuego), Magellanic moorland (southern Chile and Argentina, limited by the Magellanic forest province to the east), Malvinas islands (Argentinean archipelago of the Malvinas or Falkland islands and South Georgia island, situated in the south Atlantic ocean, about $550 \mathrm{~km}$ from Tierra del Fuego), and Juan Fernández islands (Chilean islands of Masatierra or Robinson Crusoe, Masafuera or Alejandro Selkirk, and Santa Clara, situated in the Pacific ocean, $600 \mathrm{~km}$ west of Valparaiso, at $33^{\circ}$ south latitude).
\end{abstract}

Key words: Subantaretic, biogeography, South America.

The Subantarctic subregion basically comprises the austral Andes and several small islands of southern Argentina and Chile (Morrone, 1996d, 1999). Its remarkable biota has attracted the attention of several authors. Some of them (Monrós, 1958; Kuschel, 1960; Cabrera \& Willink, 1973; Cabrera, 1976; Crisci et al., 1991; Morrone, 1996c, d) emphasized the distinctiveness of the Subantarctic biota and its close relationship with the Australasian biota. Cabrera (1976) listed several plant genera distributed in the Subantarctic subregion as well as in Australia, New Zealand, and/ or New Guinea, namely, Abrotanella, Aristotelia, Astelia, Carpha, Donatia, Drapetes, Eucryphia, Gaimardia, Laurelia, Lomatia, Luzuriaga, Marsippospermum, Nothofagus, Oreobolus, Orites, Phyllacne, Pseudopanax, Rostkowia, Schoenus, Selliera, Tetrachondra, and Veroni. $c a$.

Within South America, the Subantarctic subregion has been placed in the Andean region (Morrone, 1999), where it is considered to be most closely related to the Central Chilean subregion (Morrone, 1994b; Morrone et al., 1997; Troncoso \& Romero, 1998). In addition, this subregion shows also some biotic relationships with the Parana subregion of the Neotropical region (Kuschel, 1960; Cabrera, 1976; Morrone \&
Lopretto, 1994; Maury et al., 1996), which cauld evidence a former connection between these areas.

My objective is to formalize a new biogeographic scheme for the Subantarctic subregion, providing the taxa endemic or characteristic to it and its provinces.

\section{MATERIAL AND METHODS}

Distributional data for this study were obtained from the literature. Names and classification of the bird and mammal taxa follow Sibley \& Monroe (1990) and Nowak (1991), respectively. For the subregion and each province, the distribution of an endemic taxon is represented as an individual track (see Morrone \& Crisci, 1995; Craw et al., 1999).

\section{RESULTS}

\section{Subantarctic subregion}

(Figs. 1, 2)

The Subantarctic subregion (Morrone, 1999) comprises the austral Andes, from $37^{\circ}$ south latitude to Cabo de Hornos, including the archipelago of southern Chile andArgentina, the Malvinas or Falkland islands, South Georgia island, and Juan Fernández islands. It comprises 
six provinces (Fig. 1). The track of Germainiellus (Fig. 2) is representative of the subregion.

Symonyms. Moist Andean zone (Shannon, 1927: 3), Chilean district (Cabrera \& Yepes, 1940: 16), Subantarctic forests (Soriano, 1950: 33), Subantaretic province (Cabrera, 1951: 23, 1953 : 107, 1958: 200, 1971: 37, 1976: 72; Cabrera \& Willink, 1973: 97; Morrone, 1994b: 191, 1996c: 106; Posadas et al., 1997: 2; Carpintero, 1998: 148; Roig, 1998: 140), Australcordilleran domain (Ringuelet, 1955b: 84), Subantarctic forests region (Hueck, 1957: 40), Araucanian subregion (Monrós, 1958: 145; Ringuelet, 1961: 156; Rapoport, 1968: 75), Austral-cordilleran domain (Ringuelet, 1961: 160), Andean-Patagonian forests (Ragonese, 1966: 35), Chilean province (Fittkau, 1969: 642), SouthernAndes (Sick, 1969: 465), Subantarctic domain (Cabrera, 1971: 36; Cabrera \& Willink, 1973: 96; Cabrera, 1976: 71), Nothofagus centre (Müller, 1973: 155), Patagonian province (Ringuelet, 1975: 107), Andean Subantarctic region (Rivas Martínez \& Tovar, 1983:521), Chilean subregion (Flint, 1989: 1), Valdivian-Magellanian region (RivasMartínez \& Navarro, 1994: map), Subantarctic area (Coscarón \& Coscarón-Arias, 1995: 726), Altoandean province (Roig, 1998: 139), and Subantarctic subregion (Morrone, 1999: 13).

Vegetation. Forests with dominant species belonging to the Austral genera Nothofagus, Dacrydium, Saxegothaea, Austrocedrus, Pilgerodendron, and Fitzroya (Rothkügel, 1916; Skottsberg, 1921, 1960; Cabrera, 1971, 1976; Cabrera \& Willink, 1973). There are also moorlands and other areas lacking Nothofagus, e.g., the Juan Fernández, South Georgia, and Malvinas islands. Inclusion of the latter within the Subantarctic subregion, however, was postulated by Ringuelet (1955a) and supported by more recent cladistic biogeographic analyses (Morrone, 1993a; Morrone et al., 1994; Posadas et al., 1997).

Taxa. FUNGI. Cyttariales. Cyttariaceae: Cyttaria darwinii, C. berteroi, C. hookeri, C. hariotii, C. espinosae, C. johowii, and C. exigua (Humphries et al., 1986; Crisci et al., 1988; Seberg, 1991; Roig-Juñent, 1994b). CONIFEROPHYTA. Coniferopsida. Coniferales. Cupressaceae: Pilgerodendron (Covas, 1995c); Podocárpaceae: Prumnopitys andina (Covas, 1995a). MAGNOLIOPHYTA. Liliopsida. Cyperales. Cyperaceae: Carpha, Oreobolus, and Schoenus (Cabrera, 1976). Juncales. Juncaceae: Marsippospermum and Rosthowia (Cabrera, 1976). Liliales. Liliaceae: Astelia, Luzuriaga, and Philesia (Cabrera, 1976). Restionales. Centrolepidaceae: Gaimardia (Cabrera, 1976). Magnoliopsida. Apiales. Apiaceae: Azorella filamentosa, A.lycopodioides, and A. selago (Martínez,
1989); Araliaceae: Pseudopanax (Cabrera, 1976). Asterales. Asteraceae: Abrotanella, Eriocaulon, Leucheria coerulescens, $L$. diemii, L. magna, Macrachaenium, Perezia nutans, and Triptilion achillae (Cabrera, 1976; Katinas et al., 1992; Katinas, 1995). Campanulales. Donatiaceae: Donatia (Cabrera, 1976). Fagales. Nothofagaceae: Nothofagus (Cranwell, 1964; Fleming, 1964; Humphries, 1981; Humphries \& Parenti, 1986; Humphries et al., 1986; Grehan, 1991; Seberg, 1991; Hill \& Jordan, 1993; Linder \& Crisp, 1995). Laurales. Gomortecaceae: Gomorteca (Cabrera, 1976); Monimiaceae: Laurelia (Cabrera, 1976). Malvales. Elaeocarpaceae: Aristotelia chilensis (Coode, 1984). Myrtales. Myrtaceae: Tepualia (Cabrera, 1976); Onagraceae: Epilobium australe, E. nivale, E. puberulum, and Fuchsia magellanica (Solomon, 1982; Berry, 1989); Thymeleaceae: Drapetes (Cabrera, 1976). Proteales. Proteaceae: Embothrium and Orites (Cabrera, 1976; Weston \& Crisp, 1987, 1994, 1996). Restionales. Restionaceat (E1lsmore, 1991). Rosales. Eucryphiaceae: Eucryphic (Cabrera, 1976). Santalales. Misodendraceae: Misodendrum (Orfila, 1978; Zavaro et al., 1997). Scrophulariales. Scrophulariaceae: Veronica (Cabrera, 1976). ANNELIDA Hirudinea. Glossiphoniiformes. Glossiphoniidae: Glossiphonia mesembrina and Helobdella scutifera (Ringuelet, 1985). Hirudiniformes. Americobdellidae: Americobdella (Ringuelet, 1968); Semiscolecidae: Patagoniobdella (Ringuelet, 1985). ARTHROPODA. Arachnida. Araneae. Anyphaenidae: Acanthoceto cinereus, A. pichi, Aporatea, and Ferreria (Gerschman de Pikelin \& Schiapelli, 1975; Ramírez, 1997); Dipluridae: Scotinoecus (Schiapelli \& Gerschman de Pikelin, 1968); Mimetidae: Gnolus (Platnick, 1993). Opiliones. Caddidae: Austropsopilio (Cokendolpher \& Maury, 1990). Pseudoscorpionida. Chthoniidae: Austrochthonius chilensis (Vitali-Di Castri, 1975); Vachoniidae: Beierobisium (Vitali-Di Castri, 1970). Chilopoda. Geophilomorpha. Geophilidae: Schendyloides (Pereira et al., 1997; Pereira, 1998). Crustacea. Anomopoda. Ilyocryptidae: Ilyocryptus brevidentatus (Paggi, 1998). Copepoda. Canthocamptidae: Attheyella crenulata (Menu Marque \& Bosnia, 1986). Decapoda. Aeglidae: Aegla alacalufi and A. denticulata (Morrone \& Lopretto, 1994; Morrone, 1996b). Hexapoda. Coleoptera. Attelabidae: Minurus (Morrone \& Posadas, 1998); Belidae: Atractuchus, Dicordylus, and Trichophtalmus (Kuschel, 1959; Vanin, 1976; Morrone \& Roig-Juñent, 1995; Morrone, 1996d); Carabidae: Antarctiola, Antarctonomus, Barypus (Arathynus), Bembidarenas, Ceroglossini, Creobius, Cascellius, Falsodromius, Merizodus, Nothanillus, Nothocascellius, Pseudomigadops, and Systolosomini (Jeannel, 1938, 1962; Straneo, 1951; Erwin, 1972; Reichardt, 1977; Roig-Juñent, 1992a, b, 1994b, 1995a, b, 1998; Morrone et al., 1994); Chrysomelidae: Stenomela (Monrós, 1958); Cleridae: Eurymetopum spp. (Solervicens, 1986, 1987; Morrone et al., 1994); Curculionidae: Antarctobius, Anthonomus ornatus species group, Aterpini, Atrichonotus pacificus, Dasydema hirtella, Falklandiellus, Falklandius generic group, 




Fig. 1. Provinces of the Subantarctic subregion.

Germainiellus, Germainius, Haversiella, and Sinophloeus (Kusche1, 1951, 1987; Wood, 1986; Clark \& Burke, 1988; Lanteri \& O'Brien, 1990; Morrone, 1992b, c, 1993a, 1994a, d, 1995, 1996a; Morrone et al, 1994; Lanteri \& Morrone, 1995; Morrone \& RoigJuñent, 1995); Dytisicidae: Lancetes (Bachmann \& Trémouilles, 1981); Nemonychidae: Nannomacer (Kuschel, 1954, 1983; Morrone, 1996d); Scarabeidae: Frickius (Zunino, 1984); Staphylinidae: Neophonus (Thayer, 1989); Tenebrionidae: Adelium spp. (Peña, 1991). Diptera. Chironomidae: Aphroteninae, Diamesinae, and Podonomopsis-Rheochlus (RoigJunent, 1994b); Ephydridae: Notiocoenia pollinosa, Scatella neglectus, and S. sturdeeanus (Mathis, 1980); Mycetophilidae: Echinopodium spp. (Duret, 1975, 1976); Mydidae: Mitrodetus (Artigas \& Papavero, 1990); Simuliidae: Cnesiamima, Gigantodax antarcticus, $G$. carmenae, $G$. femineus, $G$. igniculus species group, $G$. marginalis, G. rufescens, G. rufidulus, $G$. trifidus, and Parastrosimulium (Wygodzinky \& Coscarón, 1989; Coscarón, 1991); Tipulidae: Elnoretta, Euvaldiviana, and Valdiviana (Jong, 1989). Hemiptera. Corixidae: Sigara egbertae, S. trimaculata, and S. vuriloche (Bachmann, 1981); Eriococcidae (Humphries et al., 1986; Seberg, 1991); Peloridiidae: Pantinia spp. and Peloridium spp. (Burckhardt \& Agosti, 1991); Reduvidae: Microtomus gayi (Giacchi \& Coscarón, 1992). Hymenoptera. Diapriidae: Gladicauda (Loiácono, 1988); Formicidae: Lasiophanes (Kusnezov, 1951); Pompilidae: Chirodamus agenius and Sphictostethus (Roig-Alsina, 1985, 1987); Tiphidae: Cosila chilensis (Genise, 1984). Lepidoptera. Cossidae: Chilecomadia (Gentili, 1989; Schoorl, 1989); Hepialidae: Andeabatis, Calada, Callipielus, Dalaca, and Parapielus (Nielsen \& Robinson, 1993); Micropterigidae: Heterobathmia spp. (Kristensen \& Nielsen, 1979; Humphries et al., 1986); Noctuidae: Paraeuxoa (Jana-Sáenz, 1989; Angulo, 1990); Palaephatidae (Davis, 1986; Grehan, 1991); Pieridae:
Hypsochila argyrodile (Field \& Herrera, 1977). Neuroptera. Hemerobiidae: Conchopterella, Gayomyia, Hemerobius chilensis, $H$. nekoi, $H$. stenopterus, and Megalomus nigratus (Oswald, 1993; Monserrat, 1996, 1997). Odonata. Austropetalidae: Rheopetalia rex (Carle, 1996); Cordulidae: Rialla villosa (Muzón, 1995); Libellulidae: Sympetrum villosum (Muzón, 1995); Neopetalidae: Neopetalia punctata (Muzón, 1995); Petaluridae: Phenes r. raptor (Muzón, 1995). Orthoptera. Tristiridae: Tropidostethini (Cigliano, 1989b). Plecoptera. Amphipnoidae (Muzón \& Bachmann, 1998); Eustheniidae: Neuroperla eschendingi and Neuroperlopsis patris (Bachmann, 1995); Notonemouridae: Neofulla, Neonemura, and Udamocercia (Bachmann, 1995). Trichoptera. Hydrobiosidae (Angrisano, 1995); Hydropsychidae: Smicridea decora, S. mucronata, S. penai, and $S$. pucara (FLint, 1989), VERTEBRATA. Amphibia. Anura. Leptodactylidae: Batrachyla leptopus (Barrio, 1967a); Rhinodermatidae: Rhinoderma (Williams \& Echeverría, 1995). Aves. Anseriformes. Anatidae: Anas specularis, Chloephaga hybrida, C. poliocephala, C. rubidiceps, Tachyeres patachonicus, and T. pteneres (Navas, 1977; Olrog, 1984; Peña, 1992). Ciconiiformes. Accipitridae: Buteo ventralis (Sibley \& Monroe, 1990); Falconidae: Phalcobaenus albogularis (Olrog, 1984); Phalacrocoracidae: Phalacrocorax atriceps (Olrog, 1984); Thinocoridae: Attagis maluoinus (Olrog, 1984). Columbiformes. Columbidae: Columba aracana (Olrog, 1984). Gruiformes. Rallidae: Gallinula melanops (Navas, 1991). Passeriformes. Fringillidae: Carduelis barbata (Olrog, 1984); Furnariidae: Aphrastura spinicauda, Cinclodes antarcticus, $C$. oustaleti, Pygarrhichus albogularis, and Sylviorhynchus desmursii (Müller, 1973; Olrog, 1984); Muscicapidae: Turdus falcklandii (Olrog, 1984); Rhynocyptidae: Pteroptochus tarnii (Olrog, 1984). Psittaciformes. Psittacidae: Enicognathus ferrugineus and E. leptorhynchus (Olrog, 1984). Trochiliformes. Trochilidae: Sephanoides sephanioides (Olrog, 1984). Mammalia. Artiodactyla. Cervidae: Hippocamelus bisulcus and Pudu puda (Redford \& Eisenberg, 1992). Carnivora. Felidae: Felis guigna (Redford \& Eisenberg, 1992). Chiroptera. Vespertilionidae: Myotis chiloensis (Redford \& Eisenberg, 1992). Microbiotheria. Microbiotheriidae: Dromiciops australis (Redford \& Eisenberg, 1992). Rodentia. Muridae: Abrothrix longipilis, Auliscomys micropus, Chelemys macronyx, Euneomys chinchilloides, Geoxus valdivianus, and Irenomys tarsalis (Redford \& Eisenberg, 1992).

\section{Maule province}

(Figs. 1, 3)

Southern Chile and Argentina, between 34$37^{\circ}$ south latitude. The track of Chaetanthera serrata (Fig. 3 ) is representative of this province.

Synonyms, Araucaria forests subregion (Hueck, 1957: 40), Mountain zone (Kuschel, 1960: 545), Northern Valdivian forest region (Peña, 1966: 11), Pehueñar region (Peña, 1966: 12), Pehuén district (Cabrera, 1971: 37; Cabrera \& 
Willink, 1973: 99; Cabrera, 1976: 73), Valdivian region (O'Brien, 1971: 203), Maule district (Cabrera \& Willink, 1973: 98), Valdivian area (Artigas, 1975: map), Valdivian province (RivasMartínez \& Navarro, 1994: map), Maule (RoigJuñent, 1994b: 181), and Maule province (Morrone, 1999: 14).

Vegetation. Transitional temperate forests, with some biotic elements from the Central Chilean subregion. Dominant plant species are Acaena pinnatifida, Alstroemeria curantiaca, Aristotelia chilensis, Baccharis concava, Berberis buxifolia, Boquila trifoliata, Chusquea couleu, Cortaderia pilosa, Cryptocaria mammosa, Escallonia virgata, Lapageria rosea, Laurelia sempervicens, Myrtus luma, Nothofagus alexandri, $N$, dombeyi, $N$. leoni, $N$. obliqua, $N$. pumilio, Pernettya mucronata, Persea lingue, Podocarpus nubigena, Rhaphithammus spinosum, and Ribes magellanica (Cabrera, 1971, 1976; Cabrera \& Willink, 1973). There are small areas with forests of Araucaria araucana (Pena, 1966; Cabrera \& Willink, 1973).

TaXa. CONIFEROPHYTA. Coniferopsida. Coniferales. Araucariaceae: Araucaria araucana (Cabrera, 1976; Cabrera \& Willink, 1973; Covas, 1995b). MAGNOLIOPHXTA. Magnoliopsida. Asterales. Asteraceae: Chaetanthera linearis var taltalensis, C. serrata, and Triptilion achilleae (Morrone et al., 1997). ARTHROPODA. Arachnida. Araneae. Anyphaenidae: Acanthoceto ladormida (Ramírez, 1997); Dipluridae: Scotinoecus cinereopilosus (Schiapelli \& Gerschman de Pikelin, 1968); Gnaphosidae: Apodrassodes mercedes, A. pucon, and Echemoides malleco (Platnick, 1983; Platnick \& Shadab, 1983); Migidae: Mallecomigas (Goloboff \& Platnick, 1987); Nemestidae: Acanthogonatus brunneus, A. hualpen, A. mulchen, A. nahuelbuta, A. recinto, and $A$. tolhuaca (Goloboff, 1995). Opiliones. Triaenonychidae: Araucanobunus (Muñoz, 1973). Pseudoscorpionida. Chthonidae: Sathrochthonius pefauri (Vitali-Di Castri, 1974). Crustacea. Decapoda. Aeglidae: Aegla bahamondei, A. expansa, and A. spectabilis (Jara, 1992; Bond-Buckup \& Buckup, 1994).

Hexapoda. Coleoptera. Anthribidae: Dinocentrus signatipes (Morrone \& Roig-Junent, 1995); Belidae: Atractuchus argus, Callirhynchinus exquisitus, Dicordylus balteatus, and Oxycraspedus (Kuschel, 1959; Elgueta, 1986; Morrone \& Roig-Juñent, 1995; Morrone \& Posadas, 1998); Buprestidae: Mastogenius sulcicollis, Mendizabalia g. germaini, Pterobothris c. corrosus, and Trigonogenium angulosum ruginosum (Bellamy \& Moore, 1990; Roig-Juñent, 1994b); Carabidae: Barypus paralellus, Ceroglossus c.chilensis, C.c. temucensis, C. darwini magellanicus, C. valdiviae subnitens, Cnemalobus germaini, Cylindera chilensis, and Nothobroscus (Roig-Juñent \& Cicchino, 1989; RoigJuñent, 1990, 1994a, b; Roig-Juñent \& Ball, 1995); Caridae: Caenominurus (Kuschel, 1992; Morrone \&
Roig-Junent, 1995; Morrone \& Posadas, 1998); Cleridae: Eurymetopum frigidus, E. gayi, and E.vittula (Solervicens, 1986); Curculionidae: Aegorhinus albolineatus, A. boviei, A. nitens, A. suturalis, Anthonomus araucanus, A. chilicola, Araucarietus, Araucarius chilensis, A. major, A. medius, A. minor, Calvertius, Cylydrorhinus inaequatus, Dasydema annucella, Eisingius, Eucalus tesselatus, Geniocremnus chiliensis, Hybreoleptops aureosignatus, Lamiarhinus horridus, Listroderes brevirostris, Megalometides spp., Megalometis andigena, Nothofaginoides, Omoides validus, Planus, Polydrusus roseus, Rhyephenes gayi, $R$. lateralis, Tartarisus perforatipennis, and $T$. subfasciatus (Kuschel, 1952, 1966; May, 1967; Rühm, 1987; Clark \& Burke, 1988; Morrone, 1992a, 1996a, e; Morrone \& Roig-Juñent, 1995); Endomychidae: Chileobius cekalovici and notatus (Pakaluk \& Slipinski, 1990); Megalopodidae: Palophagoides (Kuschel \& May, 1996); Nexxonychidae: Mecomacer, Nannomacer germaini, Rhynchitomacer, and Rhynchitomacerinus (Kusche1, 1954, 1959, 1983; Morrone \& Roig-Juñent, 1995). Hymenoptera. Bradynobaenidae: Bradynobaenus australis (Genise, 1986); Formicidae: Pogonomyrmex vermiculatus (Kusnezov, 1978). Lepidoptera. Choreutidae: $N y x$ (Heppner, 1982); Cossidae: Andestana, Austrocossus, Schausisea, Surcossus, and Philanglaus (Gentili, 1989; Schoorl, 1989); Palaephatidae: Metaphatus cirrhus, M. sinuatus, Palaephatus latus, and P. leucacrotus (Davis, 1986). Odonata. Austropetaliidae: Ophiopetalia araucana and $O$. pudu (Carle, 1996). Orthoptera. Tristiridae: Elysiacris angusticollis (Cigliano, 1989a; Roig-Juñent, 1994b). Trichoptera. Hydropsychidae: Smicridea complicatissima, S. redunca, S. tregala, and S. turgida (Flint, 1989). VERTEBRATA Amphibia. Anura. Leptodactylidae: Telmatobufo bullocki (Cei, 1973). Mammalia. Rodentia. Ctenomyidae: Ctenomys maulinus (Redford \& Eisenberg, 1992); Octodontidae: Aconaemys sagei and Octodon bridgesi (Redford \& Eisenberg, 19926.

\section{Valdivian province}

(Figs. 1, 4)

Southern Chile and Argentina, south of the Maule province, reaching $47^{\circ}$ south latitude. The track of Crinodendron hookerianum (Fig. 4) is representative of this provinee.

Synonyms. Valdivian forest (Soriano, 1950: 33; Kuschel, 1960: 541; Roig-Juñent, 1994b: 181), Subantarctic forests subregion (Hueck, 1957: 40), Mountain zone (Kuschel, 1960: 545), Valdivian region (O'Brien, 1971: 203), Valdivian forest region (Peña, 1966: 14), Valdivian cordillera region (Peña, 1966: 15), Caducifolius forest district (Cabrera, 1971: 37, 1976: 73; Cabrera \& Willink, 1973: 100), Valdivian forest district (Cabrera, 1971: 38, 1976: 75; Cabrera \& Willink, 1973: 98; Roig, $1998: 140$ ), Aysenian district (Cabrera, 1971: 37), Valdivian province (Rivas- 


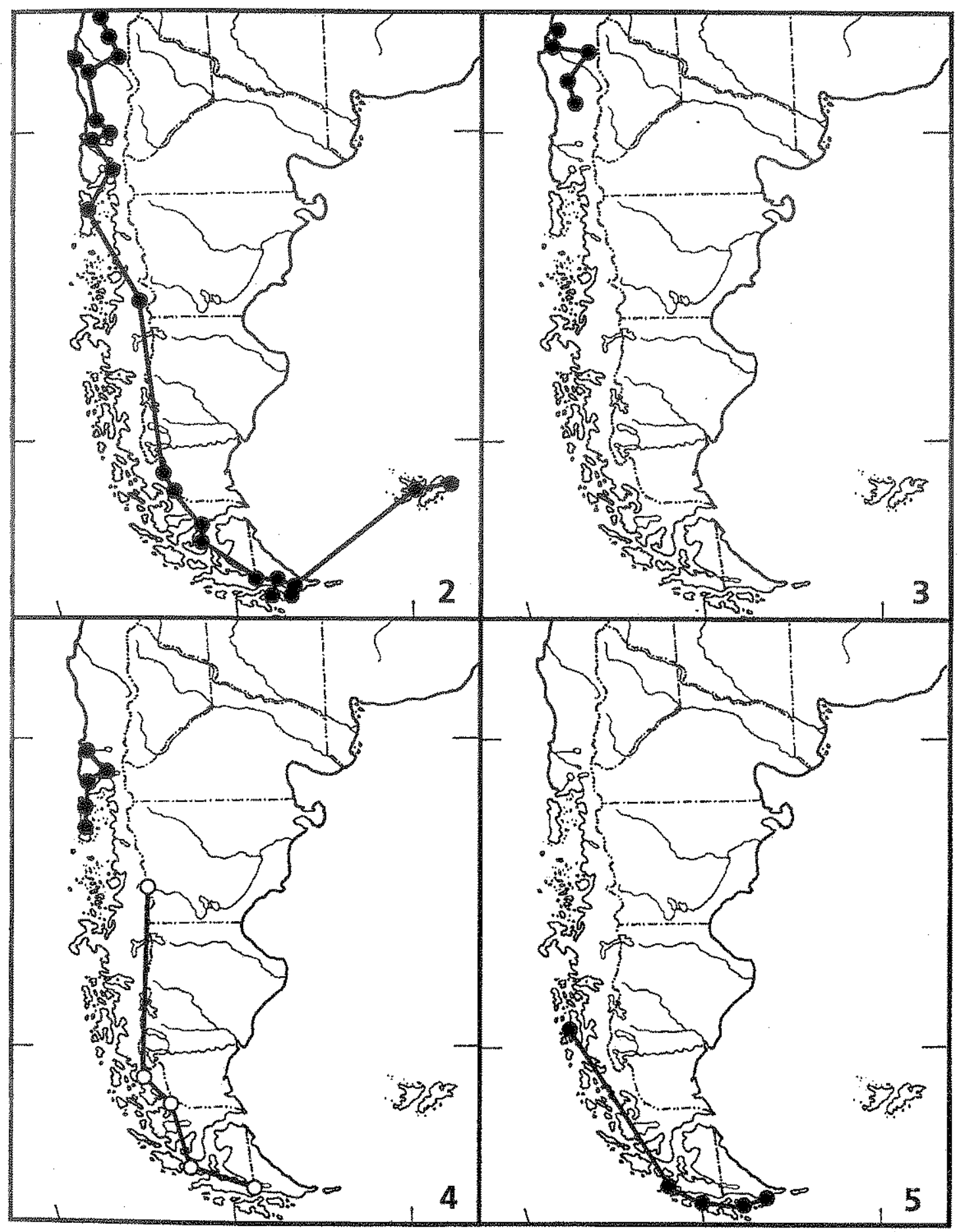

Figs. 2-5. Individual tracks. 2, Subantarctic subregion (Germainiellus); 3, Maule province (Chaetanthera serrata); 4 , black circles, Valdivian province (Crinodendron hookerianum), open circles, Magellanic forest province (Gigantodax briophyi); 5, Magellanic moorland province (Notocascellius hyadesii). 
Martínez \& Navarro, 1994: map; Morrone, 1999 : 14), and Valdivian temperate forests ecoregion (Dinerstein et $a l$. 1995: 101).

Vegetation. Temperate forests (Cabrera \& Willink, 1973). Dominant plant species are Anemone multifida, Austrocedrus chilensis, Berberis spp., Blechnum chilense, Chrysanthemum leucanthemum, Chusquea couleu, Colletia spinosa, Coriaria ruscifolia, Dasyphyllum diacanthoides, Diostea juncea, Eucryphia cordifolia, Fabiana imbricata, Fragaria chiloensis, Fitzroya cupressoides, Gevuina avellana, Laurelia philippiana, Lomatia hirsuta, Maytenus boaria, Mutisia decurrens, M. spinosa, Myrceugenella apiculata, Nothofagus antarctica, $N$. betuloides, $N$.dombeyi, $N$. procera, $N$.obliqua, $N$. procera, N. pumilio, Podocarpus nubigenus, Scirpus californicus, Senecio microcephalus, and Weimannia trichosperma (Soriano, 1950; Cabre* ra, 1971, 1976; Cabrera \& Willink, 1973).

TaXa. CONIFEROPHYTA. Coniferopsida. Coniferales. Cupressaceae: Austrocedrus and Fitzroya (Cabrera, 1976; Covas, 1995c); Podocarpaceae: Podocarpus nubigenus and Saxegothaea (Covas, 1995a). MAGNOLIOPHYTA. Magnoliopsida. Asterales. Asteraceae: Chaetanthera brachylepis and C. elegans var. elegans (Morrone et al., 1997). Malvales. Elaeocarpaceae: Crinodendron hookerianum (Coode, 1987). Myrtales. Onagraceae: Epilobium obscurum (Solomon, 1982). Santalales. Misodendraceae: Misodendrum angulatum, $M$. brachystachyum, and $M$. gayanum (Zavaro et al., 1997). MOLLUSCA. Gastropoda. Pulmonata. Ancylidae: Gundlachia foncki (Castellanos \& Miquel, 1991). ARTHROPODA. Arachnida. Araneae. Malkaridae: Chilenodes (Platnick \& Forster, 1987); Nemesiidae: Acanthogonatus confusus, $A$. franki, and $A$. subcalpeianus (Goloboff, 1995). Opiliones. Triaenonychidae: Diasia (Maury, 1987). Crustacea. Decapoda. Aeglidae: Aegla araucaniensis, $A$. riolimayana,A.d.denticulata,A.d.lacustris, A.manni, and A. rostrata (Bond-Buckup \& Buckup, 1994; Morrone \& Lopretto, 1994). Hexapoda. Coleoptera. Anthribidae: Dinocentrus tuberculosus and Sistellorhynchus posticallis (Morrone \& Roig-Juñent, 1995); Brentidae: Apion pachymerum (Morrone \& RoigJunent, 1995); Carabidae: Ceroglossus valdiviae chiloensis, C. d. darwini, C. speciosus, Creobius eudouxy, Cylindera gormazi, Nothanillus luisae, Notaphus stenoderus, Thalassobius testaceus, Trechisibus obtusiusculus, and T. oerobates (Jeannel, 1962; Roig-Juñent, 1994b); Cleridae: Eurymetopum proteus (Roig-Juñent, 1994b; Solervicens, 1986); Curculionidae: Aegorhinus fascicularis, A. kuscheli, $A$. maestus, Anthonomus berberidis, Berberidicola crenulatus, Cnemocoelus valdivianus, Epaetius, Eucalus fasciolatus, $E$. unicolor, Falklandius chilensis, $F$.peckorum, Gayus elegans, Germainiellus attenuatus, G.punctiventris, Heteromagdalis heteronyx, Listroderes obrieni, Myelobius bioculatus, $M$. fasciolatus, Neopsilorhinus, Nothofagius, Nothofagobius, Nototactus, Pachytrogus crassirostris, Philippius, Puranius fasciculiger, Rhyephenes clathratus, Tartarisus griseus, and Wittmerius (Elgueta, 1985; Clark \& Burke, 1988; Morrone, 1990, 1993a, b, 1996a, e; Morrone \& Anderson, 1995; Morrone \& Roig.Juñent, 1995; Morrone et al., 1997); Nemonychidae: Nannomacer wittmeri (Kuschel, 1959; Morrone \& RoigJuñent, 1995); Scarabeidae: Frickius variolosus (Howden, 1982); Staphylinidae: Pseudopsis adustipennis (Herman, 1975). Diptera. Simuliidae: Gigantodax igniculus (Wygodzinky \& Coscarón, 1989); Tipulidae: Valdiviana edwarsina and $V$. shannonina (Jong, 1989). Hymenoptera. Formicidae: Anthichyhonidris bidentatus (Roig-Juñent, 1994b); Ichneumonidae: Notophrudus (Porter, 1993). Lepidoptera. Palaephatidae: Apophatus, Metaphatus ichnius, Palaephatus albiterminus, $P$. amplisaccus, $P$. fusciterminus, $P$. luteolus, $P$. nielsen,$P$. spinosus, $P$. striatus, Sesommata albimaculata, S. leuroptera, and S. paraplatysaris (Davis, 1986; Roig-Junent, 1994b). Neuroptera. Hemerobidae: Megalomus democraticus and $M$. stangei (Monserrat, 1997). Odonata. Austropetalidae: Ophiopetalia diana (Carle, 1996). Orthoptera. Acrididae: Nahuelia anthracina (Ronderos \& Turk, 1989). Trichoptera. Hydrobiosidae: Australobius (Schmid, 1989), VERTEBRATA. Amphibia. Anura. Leptodactylidae: Batrachyla antartandica, Hylorina sylvatica, and Telmatobufo australis (Barrio, 1967a, b; Cei, 1973). Mammalia. Paucituberculata. Caenolestidae: Rhyncholestes raphanurus (Redford \& Eisenberg, 1992; Contreras \& Yáñez, 1995). Rodentia. Muridae: Abrothrix sanborni (Redford \& Eisenberg, 1992).

\section{Magellanic forest province \\ (Figs. 1, 4)}

Southern Chile from $47^{\circ}$ south latitude to Cabo de Hornos, and southern Argentina in small portions of western Santa Cruz and Tierra del Fuego. The track of Gigantodax briophyi (Fig. 4) is representative of this province.

Synonyms. Magellanic forests subregion (Hueck, 1957: 40), Magellanic forest (Holdgate, 1960: 560), Valdivian forest (Kuschel, 1960:543), Aysén cordillera region (Peña, 1966: 15), Magellanes Interoceanic region (Peña, 1966: 15), Magellanic district (Cabrera, 1971: 39, 1976: 77), Magellanic region (O'Brien, 1971: 204), Magellanic district (Cabrera \& Willink, 1973: 98), Austral Forest zone (Cekalovic, 1974: 305), Shrub zone (Cekalovic, 1974: 306), Magellanic area (Artigas, 1975: map), Austroandean province (Rivas-Martínez \& Navarro, 1994: map), Pacific region (Roig-Juñent, 1994b: 182), Subpolar Nothofagus forests ecoregion (Dinerstein et al., 1995: 101), and Magellanic province (Morrone, 1999; 14). 
Vegetation. More xeric forests, with abundant Nothofagus betuloides trees, and evergreen swamp forests (Cabrera \& Willink, 1973; Dinerstein et al., 1995). Dominant plant species include Drimys winteri, Embothrium coccineum, Maytenus magellanicus, Nothofagus antarctica, $N$. betuloides, and $N$. pumilio (Rothkügel, 1916; Kuschel, 1960; Tuhkanen et al., 1990).

TaXa. CONIFEROPHYTA. Coniferopsida. Coniferales. Podocarpaceae: Lepidothamnus fonkii (Covas, 1995a). MAGNOLIOPHYTA. Magnoliopsida. Myrtales. Onagraceae: Epilobium conjugens (Solomon, 1982). ARTHROPODA. Arachnida. Araneae. Dipluridae: Scotinoecus fasciatus (Schiapelli \& Gerschman de Pikelin, 1968). Hexapoda. Coleoptera. Carabidae: Antarctonomus complanatus, Cascellius aeneoniger, C. gravesii, Notaphiellus cekalovici, Notaphus kuscheli, Notholopha atrum, Peryphus rufoplagiatus, Pseudomigadops ovalis, Trechisibus antarcticus, and T. hornesis (Jeannel, 1962; Roig-Juñent, 1994b, 1995b); Curculionidae: Antarctobius germaini, A. hyadesii, A. lacunosus, Cylydrorhinus ursinus, C. vittatus, Falklandiopsis, Germainiellus laevirostris, and G. lugens (Morrone, 1992b, 1993a; Morrone \& Anderson, 1995; Morrone \& Roig-Juñent, 1995); Perimylopidae: Ptrahelops quadricollis (Roig-Junent, 1994b). Lepidoptera. Noctuidae: Paraeuxoa perdita (Jana-Sáenz, 1989; Angulo, 1990). Neuroptera. Hemerobiidae: Megalomus australis (Monserrat, 1997). VERTEBRATA. Mammalia. Rodentia. Muridae: Abrothrix lanosus (Redford \& Eisenberg, 1992).

\section{Magellanic moorland province \\ (Figs. 1,5)}

Southern Chile and Argentina, limited by the Magellanic forest province to the east. The track of Notocascellius hyadesii (Fig. 5) is representative of this province.

Synonyms. Magellanic moorland (Godley, 1960: 467), Magellanic moorland zone (Kuschel, 1960: 544), Southern Pacific region (Peña, 1966: 16), Magellanic region (O'Brien, 1971: 204), Austral Pacific zone (Cekalovic, 1974: 301), Patagonian Ice zone (Cekalovic, 1974: 303), Magellanic area (Artigas, 1975: map), Austroandean province (Rivas-Martínez \& Navarro, 1994: map), Fuegian province (RivasMartínez \& Navarro, 1994: map), Eastern forest (Roig-Juñent, 1994b: 182), Subpolar Nothofagus forests ecoregion (Dinerstein et al., 1995: 101), and Magellanic moorland province (Morrone, 1999: 14).

Vegetation. Moorlands (Cabrera \& Willink, 1973). Dominant plant species include Astelia pumila, Donatia fascicularis, Drimys winteri, Gaimardia australis, Hymenophyllum spp.,
Nothofagus betuloides, N. pumilio, Oreobolus obtusangulus, Schoenus antarcticus, and Sphagnum magellanicum (Kuschel, 1960; Tuhkanen et al., 1990).

Taxa. ARTHROPODA. Hexapoda. Coleoptera. Brentidae: Apion fuegianum (Cekalovic, 1974); Carabidae: Ceroglossus s. suturalis, Feroniomorpha lucida, Metius malachitichus, Mimodromius nigrotestaceus, and Notocascellius hyadesii (RoigJuñent, 1994b, 1995b); Caridae: Chilecar (Kuschel, 1992; Morrone \& Roig-Juñent, 1995); Curculionidae: Aegorhinus vitulus, Antarctobius rugirostris, A. yefacel, Berberidicola exaratus, and Telurus (Cekalovic, 1974; Morrone, 1992b; Morrone \& Anderson, 1995; Morrone \& Roig-Juñent, 1995). Diptera. Chironomidae: Parochlus pilosus (Roig-Juñent, 1994b); Scatopsidae: Diamphidicus chilensis (Amorim, 1990); Simuliidae: Gigantodax antarcticus, $G$, brophyi, and G. rufidulus (Roig-Junent, 1994b). VERTEBRATA. Mammalia。 Rodentia. Muridae: Abrothrix hershkovitzi and $A$. markhami (Redford \& Eisenberg, 1992).

\section{Malvinas islands province}

Argentinean archipelago of the Malvinas or Falkland islands and South Georgia island, situated in the south Atlantic Ocean, abott 550 $\mathrm{km}$ from Tierra del Fuego.

Synonyms. Insular province (Cabrera, 1951: 23, 1953: 107, 1958: 200, 1971: 40; Cabrera \& Willink, 1973: 102; Cabrera, 1976: 78), Patagonian grasslands ecoregion (Dinerstein et al., 1995: 108), Cortaderal Malvinian district (Roig, 1998: 142), Tundra or Georgias district (Roig, 1998: 142), and Mavinas islands province (Morrone, 1999: 14).

Vegetation. Grasslands, steppes, and tundra (Cabrera \& Willink, 1973; Dinerstein et al, 1995). Dominant plant species are Abrotanella emarginata, Acaena adscendens, A. magellanica, A. microcephala, Astellia pumila, Azorella lycopodioides, A. selago, Baccharis magellanica, Blechnum tabulare, Bolax gummifera, Caltha appendiculata, Carex trifida, Cortaderia pilosa, Deschampsia flexuosa, Empetrum rubrum, Festuca erecta, Gaimardia australis, Juncus sheuzerioides, Pernettya pumila, Poa annua, $P$. antarctica, P. flabellata, Pratia repens, Senecio candicans, $S$. littoralis, S. vaginata, Uncinia smithit, and Veronica elliptica (Skottsberg, 1921; Davies, 1939; Moore, 1968; Cabrera, 1971, 1976; Cabrera \& Willink, 1973).

Taxa. MAGNOLIOPHYTA. Magnoliopsida. Asterales. Asteraceae: Chevreulia lycopodioides, Gamochaeta affinis, Nassauvia gaudichaudii, $N$. serpens, and Senecio littoralis (Cabrera, 1.976; Katinas, 1995). Ranunculales. Ranunculaceae: Ranunculus acaulis (Moore, 1968). PLATYHELMINTHES. Turbelaria. Dugesiidae: Neppia falklandica 
(Cazzaniga, 1995). ANNELIDA. Hirudinea. Glossiphoniiformes. Glossiphoniidae: Adaetobdella malvinensis (Ringuelet, 1985; Lopretto, 1995a). ARTHROPODA. Chilopoda. Geophilomorpha. Schendylidae: Schendyloides alacer (Pereira \& Minelli, 1992). Crustacea. Amphipoda. Gammaridae: Falklandella and Praefalklandella (Stock \& Platvoet, 1991; Lopretto, 1995b); Hyallelidae: Hyalella neonoma (Stock \& Platvoet, 1991). Anostraca. Branchinectidae: Branchinecta gaini (Cohen, 1998). Hexapoda. Coleoptera. Byrrhidae: Chalcosphaerium solox and $C$. enderleini (Voisin, 1987); Carabidae: Pseudomigadops darwini, and $P$. falklandicus (Jeanne1, 1938, 1962; Roig-Juñent, 1994b); Curculionidae: Antarctobits abditus, A. bidentatus, A. falklandicus, Caneorhinus biangulatus, C. uretai, Cylydrorhinus lemniscatus, Falklandius goliath, $F$. kuscheli, $F$. turbificatus, Germainiellus salebrosus, Lanteriella, Malvinius, Puranius championi, P. exsculpticollis, and P. scaber (Robinson, 1984; Voisin, 1987; Morrone, 1992b, c, 1993a, 1994c; Morrone \& Anderson, 1995; Morrone \& Roig-Juñent, 1995); Leiodidae: Falkocholeva falklandica and Falkonemadus sphenisci (Robinson, 1984; Voisin, 1987); Perimylopidae: Darwinella amarodies, Parahelops falklandicus, $P$. haversi, and P. quadricollis (Voisin, 1987; Flores, 1998); Pythidae: Poophylax falklandica (Voisin, 1987). Diptera. Ephydridae: Scatella neglecta and S. sturdeana (Lizarralde de Grosso, 1998). Orthoptera. Gryllacrididae: Parudenus falklandicus (Robinson, 1984).VERTEBRATA. Aves. Anseriformes. Anatidae: Anas g. georgica, Chloephaga picta leucoptera, and Tachyeres brachypterus (Navas, 1977; Olrog, 1984). Ciconiiformes. Falconidae: Phalcobaenus australis (Olrog, 1984). Mammalia. Carnivora. Canidae: Dusicyon australis (Nowak, 1991).

\section{Juan Fernández islands province}

Chilean islands of Masatierra (= Robinson Crusoe), Masafuera (= Alejandro Selkirk), and Santa Clara, situated in the Pacific Ocean, 600 $\mathrm{km}$ west of Valparaiso, at $33^{\circ}$ south latitude.

Synonyms. Juan Fernández province (Cabrera \& Willink, 1973: 103), Fernandezian region (Takhtajan, 1986: 252), and Juan Femández islands province (Rivas-Martínez \& Navarro, 1994: map; Morrone, 1999: 14).

Vegetation. Forests, shrublands, and grasslands (Cabrera \& Willink, 1973). Dominant plant species include Azara fernandeziana, Coprosma hookeri, Diksonia fernandeziana, Drimys confertifolia, Dysopsis hirsuta, Empetrum rubrum, Escallonia callcottiae, Fagara mayu, Juania australis, Myrceugenia schulzei, Pernettya rigida, Rhaphithamnus venustus, Robinsonia gayana, R. gracilis, and Ugni selkirkii (Cabrera \& Willink, 1973; Stuessy et al., 1984).

Taxa. BRYOPHYTA. Bartramiaceae: Breutelia masafuerae and Philonis glabrata (Mahú, 1995);
Bryaceae: Bryum fernandezianum (Mahú, 1995); Cryphaeaceae: Cyptodon crassinervis (Mahú, 1995); Dicranaceae: Campylopus aberrans, C. blindioides, Dicranella costata, and Dicranoloma capillifolioides (Mahú, 1995); Ditrichaceae: Astomiopsis pacifica (Mahú, 1995); Fissidentaceae: Fissidens crassicuspes and $k$. fernandezianum (Mahú, 1995); Hookeriaceae: Acrophyllum tenuinerve (Mahú, 1995), Distichophy. llum subelimbatum (Mahú, 1995); Neckeraceae: Neckera rotundata, Pinnatella microsticta, and Thamnobryum caroli (Mahú, 1995); Orthotricaceae: Vlota fernandeziana (Mahú, 1995); Ptychomitriaceae: Ptychomitrium fernandezianum (Mahú, 1995); Racopilaceae: Racopilum fernandezianum (Mahú, 1995); Thuidaceae: Thuidium masafuerae (Mahú, 1995). FLICOPHYTA. Ficopsida. Filicales. Dicksoniaceae: Dicksonia berteriana (Murillo, 1988). MAGNOLIOPHYTA. Liliopsida. Arecales. Arecaceae: Juania australis (Cabrera \&Willink, 1973). Magnoliopsida. Asterales. Asteraceae: Centaurodendron, Dendroseris, Phaenicoseris spp., Rea, Rhetinodendron, Robinsonia, and Yunquea (Cabrera \& Willink, 1973; Stuessy et al., 1984, 1990; Sanders et al., 1987; Crawford et al., 1992). Campanulales. Campanulaceae: Wahlenbergia fernandeziana complex (Lammers, 1996). Caryophyllales. Chenopodiaceae: Chenopodium sanctaeclarae (Stuessy et al, 1990). Euphorbiales. Euphorbiaceae: Dysopsis hirsuta (Stuessy et al., 1984). Magnoliales. Lactoridaceae (Stuessy et al., 1984). Myrtales. Myrtaceae: Ugni selkirkii (Stuessyet al., 1984). Verbenales. Verbenaceae: Rhaphitamnus venustus (Rodríguez et al., 1983). Violales, Flacourtiaceae: Azara serrata (Stuessy et al., 1984). ARTHROPODA. Hexapoda. Archaeognatha. Kuschelochilis (Wygodzinsky, 1967; Camousseight, 1995a). Coleoptera. Carabidae: Trachysarus (Straneo \& Jeanne1, 1955); Curculionidae: Anolethrus, Juanorhinini, Pachystylus, and Strongylopterus ovatus (Kuschel, 1952; Wibmer \& O’Brien, 1986). Diptera. Simuliidae: Gigantodax kuscheli (Wygodzinky \& Coscarón, 1989). Neuroptera. Hemerobiidae: Conchopterella kuscheli and C. maculata (Oswald, 1993). Thysanoptera. Thripidae: Physothrips skottsbergi (Prado \& Muñoz, 1995). Thysanura. Lepismatidae: Isolepisma annectens. (Camousseight, 1995b).

\section{ACKNOWLEDGMENTS}

I am grateful to Paula Posadas and Sergio Roig-Juñent for critically commenting on a preliminary draft of the manuscript.

\section{BIBLIOGRAPHY}

Amorim, D. S. 1990. A new species of the genus Diamphidicus Cook (Diptera, Bibionomorpha, Scatopsidae) from Chile. Rev. Bras. Entomol. 33(3/ 4): $477-482$.

Angrisano, E. B. 1995. El orden Trichoptera en la Argentina y países limitrofes. Physis (Buenos Aires) 
B, 50(118-119): 19-25.

Angulo, A. 1990. Paraeuxoa Forbes, 1933, versus Caphornia Koehler, 1958 (Lepidoptera: Noctuidae): Sizonimia de dos géneros andino-patagónicos. Rev. Chil. Entomol. 18: 13-17.

Artigas, J. N. 1975 . Introducción al estudio por computación de las áreas zoogeográficas de Chile continental basado en la distribución de 903 especies de animales terrestres. Gayana, misc. 4:1-25.

Artigas, J. N. \& N. Papavero. 1990. Studies of Mydidae (Diptera). V. Phylogenetic and biogeographic notes, key to the American genera and illustrations of spermathecae. Gayana Zool. 54(3-4): 87-116.

Bachmann, A. O. 1981. Insecta. Hemiptera. Corixidae. In: Ringuelet, R. A. (dir.), Fauna de Agua Dulce de la República Argentina, Fecic, Buenos Aires, $35(2)$ : $1-270$.

1995. Insecta Plecoptera. In: Lopretto, E. C. \& G. Tell (eds.), Ecosistemas de aguas continentales: Metodologías para su estudio, III, Ediciones Sur, La Plata, pp. 1093-1111.

Bachmann, A. O \& E. R. Trémouiles. 1981. El género Lancetes en la Argentina (Coleoptera, Dytiscidae). Physis (Buenos Aires) B, 39(97):103-1.18.

Barrio, A. 1967a. Batrachyla antartandica n. sp. (Anura, Leptodactylidae): Descripción y estudio comparativo con la especie genotípica, $B$. leptopus Bell. Physis (Buenos Aires) 27(74): 101-109.

- 1967b. Observaciones etoecológicas sobre Hylorina sylvatica Bell (Anura, Leptodactylidae). Physis (Buenos Aires) 27(74): 153+157.

Bellamy, C. L. \& T. Moore. 1990. A review of the tribe Mendizabalini (Coleoptera: Buprestidae). Rev. Chil. Entomol. 18: 39-48.

Berry, P. E. 1989. A systematic revision of Fuchsia sect. Quelusia (Onagraceae). Ann. Missouri Bot. Gard. 76(2): 532-584.

Bond-Buckup, G. \& L. Buckup. 1994. A famítia Aeglidae (Crustacea, Decapoda, Anomura). Arq. Zool. Sâo Paulo, 32(4): 159-346.

Burckhardt, D. \& D. Agosti. 1991. New records of South American Pelorididae (Homoptera: Coleorhyncha). Rev. Chil. Entomol. 19:71-75.

Cabrera, A. \& J. Yepes. 1940. Mamiferos sud-americanos (vida, costumbres y descripción). Historia Natural Ediar, Buenos Aires.

Cabrera, A. L. 1951. Territorios fitogeográficos de la República Argentina. Bol. Soc. Argent. Bot. 4(1-2): 21-65.

- 1953. Fsquema fitogeográfico de la República Argentina. Rev. Mus. La Plata (N. S.), Bot. 8(33): 87168.

- 1958. Fitogeografía en la Argentina. Suma Geogr. 3: 101-207.

- 1971. Fitogeografía de la República Argentina. Bol. Soc. Argent. Bot. 14(1-2): 1-42.

- 1976. Regiones fitogeográficas argentinas. In: Kugler, W. F. (ed.), Enciclopedia Argentina de Agricultura y Jardineria, II, ACME, Buenos Aires, pp. $1-85$.

Cabrera, A. L. \& A. Willink. 1973. Eingeografia de América Latina. Monografía 13, Serie de Biologia, OEA, Washington D.C.

Camousseight, A. 1995a. Archaeognatha. In: Simonetti,
J. A., M. T. K. Arroyo, A. E. Spotorno \& E. Lozada (eds.), Diversidad biológica de Chile, Conicyt, Santiago de Chile, pp. 214-215.

- 1995b. Thysanura. In: Simonetti, J. A., M. T. K. Arroyo, A. E. Spotorno \& E. Lozada (eds.), Diversidad biológica de Chile, Conicyt, Santiago de Chile, pp. 216-217.

Carle, F. L. 1996. Revision of Austropetalidae (Anysoptera: Aexhnoidea). Odonatologica 25(3): $231-259$.

Carpintero, D. L. 1998. Miridae. In: Morrone, J. J. \& S. Coscarón (eds.), Biodiversidad de artrópodos argentinos: Un enfoque biotaxonômico, Ediciones Sur, La Plata, pp. 144-150.

Castellanos, Z. A. de \& S. E. Miquel. 1991. Distribu ción de los Pulmonata Basommatophora. In: Castellanos, Z. A. de (ed,), Fauna de Agua Dulce de la Republica Argentina, Fecic, Buenos Aires, 15(9): 1-11.

Cazzaniga, N. J. 1995. Platyhelminthes Tricladida. In: Lopretto, E. C. \& G. Tell (eds.), Ecosistemas de aguas continentales: Metodologías para su estudio, II, Ediciones Sur, La Plata, pp. 619-639.

Cei, J. M. 1973. Sobre la importancia de un reciente hallazgo herpetológico en el sur de Chile. Physis (Buenos Aires) C, 32(85): 263-268.

Cekalovic, T. 1974. Divisiones biogeográficas de la XII región chilena (Magallanes). Bol. Soc. Biol. Concepción 8 8: 297-314.

Cigliano, M. M. 1989a. Revisión sistemática de la familia Tristiridae (Orthoptera, Acridoidea). Bol. Soc. Biol. Concepción 60: 51-110.

1989b. A cladistic analysis of the family Tristiridae (Orthoptera, Acridoidea). Cladistics 5: 379-393.

Clark, W. E. \& W. R. Burke. 1988. Revision of the ornatus species group of the genus Anthonomus Germar (Coleoptera: Curculionidae). Proc. Entomol. Soc. Washington 91(1): 88-111.

Cohen, R. G. 1998. Anostraca. In: Morrone, J. J. \& S. Coscarón (eds.), Biodiversidad de artropodos argentinos: Un enfoque biotaxonómico, Ediciones Sur, La Plata, pp. 491-501.

Cokendolpher, J. C. \& E. A. Maury. 1990. Austropsopilio harvestmen (Opiliones, Cyphopalpatores, Caddidae) discovered in South America. Bol. Soc. Biol. Concepción 61: 59-62.

Contreras, L. C. \& J. L. Yáñez. 1995. Mamíferos. In: Simonetti, J. A., M. T. K. Arroyo, A. E. Spotorno \& E. Lozada (eds.), Diversidad biológica de Chile, Conicyt, Santiago de Chile, pp. 336-349.

Coode, M. J. E. 1984, Aristotelia and Vallea, closely related in Elaeocarpaceae. Kew Bull. $40(3)$ : 479. 507.

- 1987. Crinodendron, Dubouzetia and Peripentadenia, closely related in Elaeocarpaceae. Kew Bull. 42(4): 777-814.

Coscarón, S. 1991. Insecta Diptera. Simuliidae. In: Castellanos, Z. A. de (ed.), Fauna de Agua Dulce de la República Argentina, Fecic, Buenos Aires, $38(2): 1-384$.

Coscarón, S. \& C. L. Coscarón-Arias. 1995. Distribution of Neotropical Simulidae (Insecta, Diptera) and its areas of endemism. Rev. Acad. Colomb. Cienc. 19(75): $717-732$. 
Covas, G. 1995a. Podocarpaceae. In: Flora Fanerogamica Argentina, fasc. 4 , no. 5a, Proflora (Conicet), Córdoba, pp. 1-6.

- 1995b. Araucariaceae. In: Flora Fanerogámica Argentina, fasc. 4, no. 6.a, Proflora (Conicet), Córdoba, pp. 7-9.

- 1995c. Cupressaceae. In: Flora Fanerogámica Argentina, fasc. 4, no. 6.b, Proflora (Conicet), Córdoba, pp. 11-14.

Cranwell, L. M. 1964. Nothofagus: Living and fossil. In: Gressitt, J. L. C. H. Lindroth, F. R. Fosberg, C. A. Fleming \& E. G. Turbott (eds.), Pacific basin biogeography: A symposium, 1963 [1964], Bishop Museum Press, Honolulu, Hawait, pp. 387-400.

Craw, R. C., J. R. Grehan \& M. J. Heads. 1999. Panbiogeography: Tracking the history of life. Oxford Biogeography Series 11, Oxford University Press, New York.

Crawford, D. J., T. F. Stuessy, M. B. Cosner, D. H. Haines, M. Silva \& M. Baeza. 1992. Evolution of the genus Dendroseris (Asteraceae: Lactuceae) on the Juan Fernandez islands: Evidence from chloroplast and ribosomal DNA. Syst. Bot. 17(4): 676-682.

Crisci, J. V., M. M. Cigliano, J. J. Morrone \& S. Roig-Juñent. 1991. Historical biogeography of southern South America. Syst. Zool. 40: 152-171.

Crisci, J. V., I. J. Gamundi \& M. N. Cabello. 1988. A cladistic analysis of the genus Cyttaria (FungiAscomycotina). Cladistics 4: 279-290.

Davies, W. 1939. The grasslands of the Falkland Islands. Government Printer, Stanley, Falkland Islands, and Crown Agents for the Colonies, London, pp. 58.

Davis, D. R. 1986. A new family of Monotrysian moths from austral South America (Lepidoptera: Palaephatidae), with a phylogenetic review of the Monotrysia. Smithson. Contrib. Zool. 434: 1-202.

Dinerstein, E., D. M. Olson, D. J. Graham, A. L. Webster, S. A. Primm, M. P. Bookbinder \& G. Ledec. 1995. Una evaluación del estado de conser. vación de las ecoregiones terrestres de América Latina y el Caribe. World Bank, Washington, D.C., $135 \mathrm{pp}$.

Duret, J. P. 1975. Cuatro especies nuevas del género Echinopodium Freeman 1951 (Diptera, Mycetophilidae). Physis (Buenos Aires) C, 34(88): 75-81.

1976. Notas sobre el género Echinopodium Freeman 1951, en Chile, con la descripción de catorce especies nuevas (Diptera, Mycetophilidae). Physis (Buenos Aires) C, 35(90): 173-195.

Elgueta, M. 1985. Heteromagdalis nuevo género chileno de Magdalinae, descripción de dos nuevas especies y antecedentes del hábitat de las especies afines (Coleoptera: Curculionidae). Rev. Chil. Entomol. 12: 95-99.

- 1986. Redescubrimiento de Callirhynchinus exquisitus (Fairmaire et Germain, 1861) (Coleoptera: Curculionoidea: Belidae). Rev. Chil. Entomol. 14: 991-101.

Ellsmore, C. 1991. Expanding Earth or natural catastrophism. Cetos Publishing, llford, pp. 203.

Erwin, T. L. 1972. Two new genera of Bembidine carabid beetles from Australia and South America with notes on their phylogenetic and zoogeographic significance (Coleoptera). Breviora 383: 1-19.

Field, W. D. \& J. Herrera. 1977. The pierid butterflies of the genera Hypsochila Wreta, Phulia Herrich Schäffer, Infraphulia Field, Pierphulia Field, and Piercolias Staudinger. Smithson. Contrib. Zool. 232: 1-64.

Fittkau, E. J. 1969. The fauna of South America. $I n$ : Fittkau, E. J. J. Lllies, H. Klinge, G. H. Schwabe \& H. Sioli (eds.), Biogeography and ecology in South America, 2, Junk, The Hague, pp. 624-650.

Fleming, C. A. 1964. Paleontology and southern biogeography. In: Gressitt, J. L. C. H. Lindroth, F. R. Fosberg, C. A. Fleming \& E. G. Turbott (eds.), Pacific basin biogeography: A symposium, 1963 [1964], Bishop Museum Press, Honolulu, Hawaii, pp. 369-385.

Flint, O. S. 1989. Studies of Neotropical caddisflies, XXXIX: The genus Smicridea in the Chilean subregion (Trichoptera: Hydropsychidae). Smithson. Contrib. Zool. 472: 1-45.

Flores, G. E. 1998. Perimylopidae. In: Morrone, J. J. \& S. Coscarón (eds.), Biodiversidad de artrópodos argentinos: Un enfoque biotaxonómico, Ediciones Sur, La Plata, pp. 241-243.

Genise, J. F. 1984. Las Anthoboscinae neotropicales y comentarios sobre la diversidad de la subfamilia (Hymenoptera, Tiphidae). Rev. Soc. Entomol. Argent. 43(1-4): 195-220.

- 1986. Las Bradynobaenidae y algunas modificaciones a la clasificación general de Hymenoptera Aculeata. Physis (Buenos Aires) C, 44(106): 39-53.

Gentili, P. 1989. Revisión de los Cossidae (Lep.) de la Patagonia andina. Rev. Soc. Entomol. Argent. 45(1-4): 3-75.

Gerschman de Pikelin, B. S. \& R. D. Schiapelli. 1975. El género Aporatea Simon, 1897 (Araneae, Anyphaenidae), Physis (Buenos Aires) C, 34(89): 183-186.

Giacchi, J. C. \& M. del C. Coscarón. 1992. Revisión de la subfamilia Microtominae. IV. Microtomus gayi (Spinola, 1852) y $M$. pessoai Lent y Suárez, 1956 (Heteroptera: Reduviidae). Physis (Buenos Aires) C, 47(113): 67-71.

Godley, E. J. 1960. The botany of southern Chile in relation to New Zealand and the Subantarctic. Proc. Roy. Soc. London, Ser. B-Biol. Sci. 152: 457475.

Goloboff, P. A. 1995. A revision of the South American spiders of the family Nemesiidae (Araneae, Mygalomorphae). Part I: Species from Peru, Chile, Argentina, and Uruguay. Bull. Am. Mus. Nat. Hist. 224: 1-189.

Goloboff, P. A. \& N. I. Platnick. 1987. A review of the Chilean spiders of the superfamily Migoidea (Araneae, Mygalomorphae).Am. Mus. Novit. 2888: 1.15.

Grehan, J. R. 1991. A panbiogeographic perspective for pre-Cretaceous Angiosperm-Lepidoptera coevolution. Aust. Syst. Bot. 4: 91-110.

Heppner, J. B. 1982. Millierinae, a new family of Choreutidae, with new taxa from Chile and the United States (Lepidoptera: Sesioidea). Smithson. 
Contrib. Zool. 370: 1-27.

Herman, L. H. 1975. Revision and phylogeny of the monogeneric subfamily Pseudopsinae for the world (Staphylinidae, Coleoptera). Bull. Amer. Mus. Nat. Hist. $155(3): 243-317$.

Hill, R. S. \& G. J. Jordan. 1993. The evolutionary history of Nothofagus. Aust. Syst. Bot. 6: 111-126.

Holdgate, M. W. 1960. Vegetation and soils in the south Chilean islands. J. Ecol. 49(3): 559 580.

Howden, H. F. 1982. Larval and adult characters of Frickius Germain, its relationship to the Geotrupini, and a phylogeny of some major taxa in Scarabeoidea (Insecta: Coleoptera), Can J. Zool. 60(11): 2713.2724 .

Hueck, K. 1957. Las regiones forestales de Sur América. Bol. Inst. Forest. Latinoam. Invest. Capac. (Mérida) 2: 1-40.

Humphries, C. J. 1981. Biogeographical methods and the southem beeches (Fagaceae: Nothofagus). In: Funk, V. A. \& D. R. Brooks (eds.), Advances in cladistics, 1, Proceedings of the First Meeting of the Willi Hennig Society, New York Botanical Garden, Bronx, pp. 177-207.

Humphries, C. J., J. M. Cox \& E. S. Nielsen. 1986. Nothofagus and its parasites: A cladistic approach to coevolution. In: Stone, A. R. \& D. L. Hawksworth (eds.), Coevolution and systematics, Clarendon Press, Oxford , pp. 55-76.

Humphries, C. J. \& L. R. Parenti. 1986. Cladistic biogeography. Clarendon Press, Oxford.

Jana-Sáenz, C. 1989. Estudio crítico del género austral Caphornia Koehler, 1958 (Lepidoptera: Noctuidae). Gayana, Zool. 53(3): 77-111.

Jara, C. G. 1992. Aegla expansa, new species (Crustacea: Decapoda: Anomura: Aeglidae) from the lower Bí-Bio river basin, Concepción, Chile. Gayana, Zool. 56(1-2): 49-57.

Jeannel, R. 1938. Les Migadopides (Coleoptera, Adephaga), une ligne subantarctique. Rev. Fr. Entomol. 5(1): 1-55.

- 1962. Les Trechidae de la Paleantarctide occidentale. In: Delamare Debouteville \& E. H. Rapoport (eds.), Biologie de l'Amerique Australe, 3, CNRS, Paris, pp. 527-655.

Jong, H. de. 1989. A revision of the South American taxa Elnoretta Alexander, Euvaldiviana Alexander and Valdiviana Alexander (Diptera: Tipulidae), with a discussion on their phylogeny. Syst. Entomol. 14: 243-257.

Katinas, L. 1995. Tribu XII. Mutisieae Cass. In: Flora Fanerogámica Argentina, fasc. 13 , no. 280, parte 1, Proflora (Conicet), Córdoba, pp. 1-58.

Katinas, L., J. V. Crisci \& S. E. Freire. 1992. Revisión sistemática y análisis cladístico del género Triptilion Ruiz et Pavón (Asteraceae, Mutiseae). Bol. Soć. Biol. Concepción 63: 101-132.

Kristensen, N. P. \& E. S. Nielsen. 1979. A new family of micropterigid moths from South America: A contribution to the morphology and phylogeny of the Micropterigidae, with a generic catalogue of the family (Lepidoptera: Zeugloptera). Steenstrupia 5: $69-147$.

Kuschel, G. 1951. La subfamilia Aterpinae en América. Reu. Chil. Entomol. 1: 205-245.
1952. Los Curculionidae de la cordillera chilenoargentina (1a. parte) (Aporte 13 de Coleoptera Curculionidae). Rev. Chil. Entomol. 2: 229-279. 1954. La familia Nemonychidae en la región Neotropical (aporte 15 de Coleoptera Curculionoidea). Rev. Chil. Hist. Nat. 9: 97-126. 1959. Nemonychidae, Belidae y Oxycorynidae de la fauna chilena, con algunas consideraciones biogeográficas. Invest. Zool. Chil. 5: 229-271.

1960. Terrestrial zoology in southern Chile. Proc. R. Soc, London, ser. B. 152: 540-550.

- 1966. A cossonine genus with bark-beetle habits, with remarks on relationships and biogeography (Coleoptera Curculionidae). New Zealand J. Sci. $9(1): 3-29$.

- 1983. Past and present of the relict family Nemonychidae (Coleoptera, Curculionoidea). GeoJournal 7(6): 490 504.

- 1987. The subfarmily Molytinae (Coleoptera: Curculionidae): General notes and description of new taxa from New Zealand and Chile. Now Zealand J. Zool. 9: 11-29.

- 1992. Reappraisal of the Baltic amber Curculionoidea described by E. Voss. Mitt. Geol. -Paläont. Inst. Univ. Hamburg 73: 191-215.

Kuschel, G. \& B. M. May. 1996. Discovery of Palophaginae (Coleoptera: Megalopodidae) on Araucaria araucana in Chile and Argentina. New Zealand Entomol. 19: 1-13.

Kusnezov. N. 1951. "Lasiophanes" Emery en la Patagonia. Acta Zool. Lilloana 12: 89m100. 1978. Hormigas argentinas: Clave para su reconocimiento. Fund. Miguel Lillo, Miscel. 61: 1-147.

Lammers, T. G. 1996. Phylogeny, biogeography, and systematics of the Wahlenbergia fernandeziana complex (Campanulaceae: Campanuloideae). Syst. Bot. 21(3): 397-415.

Lanteri, A. A. \& J. J. Morrone. 1995. Cladistic analysis of the Naupactus leucoloma species group, Eurymetopus, and Atrichonotus (Coleoptera: Curculionidae). Rev. Soc. Entomol. Argent. 54(1-4): 99-112.

Lanteri, A. A. \& C. W. O'Brien. 1990. Taxonomic revision and cladistic analysis of Atrichonotus Buchanan (Coleoptera: Curculionidae). Trans. Am. Entomol. Soc. 116(3): 697-725.

Linder, H. P. \& M. D. Crisp. 1995. Nothofagus and Pacific biogeography. Cladistics 11(1): 5 32.

Lizarralde de Grosso, M. 1998. Ephydridae. In: Morrone, J. J. \& S. Coscarón (eds.), Biodiversidad de artrópodos argentinos: Un enfoque biotaxonómico, Ediciones Sur, La Plata, pp. 365 373.

Loiácono, M. S. 1988. Estudio preliminar del género Gladicauda Early en la República Argentina y Chile (Hymenoptera-Diapridae). Rev. Asoc. Cienc. Nat. Litoral 19(1): 39-47.

Lopretto, E. C. 1995a. Annelida Hirudinea. In: Lopretto, E. C. \& G. Tell (eds.), Ecosistemas de aguas continentales: Metodologías para su estudio, II, Ediciones Sur, La Plata, pp. 729-757.

1995b. Crustacea Eumalacostraca. In: Lopretto, E. C. \& G. Tell (eds.), Ecosistemas de aguas continentales: Metodologías para su estudio, III, Edi- 
ciones Sur, La Plata, pp. 1001-1039.

Mahú, M. 1995. Briofitas. In: Simonetti, J. A., M. T. K. Arroyo, A. E. Spotorno \& E. Lozada (eds.), Diversidad biologica de Chile, Conicyt, Santiago de Chile, pp. 53-57.

Martínez, S. 1989. El género Azorella (ApiaceaeHydrocotyloideae) en la Argentina. Darwiniana 29(1-4): 139-178.

Mathis, W. N. 1980. Studies of Ephydrinae (Diptera: Ephydridae), III: Revisions of some Neotropical genera and species. Smithson. Contrib. Zool. 303 : $1-50$.

Maury, E. A. 1987. Triaenonychidae sudamericanos. II. El género Diasia Sörensen 1902 (Opiliones, Laniatores). Physis (Buenos Aires) C, 45(109): 7484.

Maury, E. A., R. Pinto da Rocha \& J. J. Morrone. 1996. Distribution of Acropsopilio chilensis Silvestri, 1904 in southern South America (Opiliones, Palpatores, Caddidae). Biogeographica $72(3) \div 127-132$.

May, B. M. 1967. Immature stages of Curculionidae: 1. Some genera in the tribe Araucariini (Cossoninae). New Zealand J. Sci. 10:644-660.

Menu Marque, S. \& A. S. Bosnia. 1986. Sobre la identidad de Atheyella (Chapputsiella) crenulata (Mrazek, 1901)(Copepoda, Harpacticoidea). Physis (Buenos Aires) B, 44(106): 49.53.

Monrós, F. 1958. Consideraciones sobre la fauna del sur de Chile y revisión de la tribus Stenomelini (Coleoptera, Chrysomelidae). Acta Zool. Lilloana 15: $143-153$.

Monserrat, V. J. 1996. Revisión del género Hemerobius de Latinoamérica (Neuroptera, Hemerobiidae). Fragm. Entomol. (Roma) 27(2): 399-523.

- 1997. Revisión del género Megalomus de Latinoamérica (Neuroptera, Hemerobiidae). Fragm. Entomol. (Roma) 29(1): 123-206.

Moore, D. M. 1968. The vascular flora of the Falkland islands. British Antarctic Survey, Scientific reports, nro. 60, London.

Morrone, J. J. 1990. Philippius Germain, a remarkable Listroderinj from southern South America (Coleoptera: Curculionidae). Coleopts. Bull. $44(4)$ : 429-436.

- 1992a. Revision of Trachodema Blanchard with the description of an allied genus from central Chile (Insecta, Coleoptera, Curculionidae). Zool. Scr. 21(4): $417-422$.

- 1992b. Revisión sistemática y análisis cladístico del género Antarctobius Fairmaire (Coleoptera: Curculionidae). Neotropica 38(99); 3-20.

- 1992c. Revision sistemática, análisis cladístico y biogeografía histórica de los géneros Falklandius Enderlein y Lanteriella gen. nov. (Coleoptera: Curculionidae). Acta Entomal. Chil. 17: 157-174.

1993a. Revisión sistemática de un nuevo género de Rhytirrhinini (Coleoptera: Curculionidae), con un análisis biogeográfico del dominio subantártico. Bol. Soc. Biol. Concepción 64: 121-145.

- $1993 \mathrm{~b}$. Systematic revision of the costirostris species group of the weevil genus Listroderes Schoenherr (Coleoptera: Curculionidae). Trans. Amer. Entomol. Soc. 119(4): 271-315.

- 1994a. Clarification of the taxonomic status of the species formerly placed in Listroderes Schoenherr (Coleoptera: Curculionidae), with the description of a new genus. Am. Mus. Novit. 3093: 1-11.

- 1994b. Distributional patterns of species of Rhytirrhinini (Coleoptera: Curculionidae) and the historical relationships of the Andean provinces. Global Ecol. Biog. Lett. 4: 188-194.

- 1994c. Systematics, cladistics, and biogeography of the Andean weevil genera Macrostyphlus, Adioristidius, Puranius, and Amathynetoides, new genus (Coleoptera: Curculionidae).Am. Mus. Novit. 3104: 1-63.

- 1994d. Cladistic placement of the Subantarctic genus Haversiella (Coleoptera: Curculionidae). J. New York Entomol. Soc. 102(3): 299-302.

- 1995. Estudio taxonómico y biogeográfico del género subantártico Falklandiellus Kuschel (Coleoptera: Curculionidas). Physts (Buenos Aires) C, 50(118-119): 105-110.

- 1996 a. Distributional patterns of the South American Aterpini (Coleoptera: Curculionidae). Rev. Soc. Entomol. Argent. 55(1-4): 131-141.

- 1996b. On the geographical distribution of Aegla Leach: Identification of areas of endemism (Decapoda: Aeglidae). Biogeographica 72(3): 113 119.

- 1996c. The biogeographical Andean subregion: A proposal exemplified by Arthropod taxa (Arachnida, Crustacea, and Hexapoda). Neotropica 42(107-108): 103-114.

- 1996 d. Austral biogeography and relict weevil taxa (Coleoptera: Nemonychidae, Belidae, Brentidae, and Caridae). J. Comp. Biol. 1(3-4): 123-127.

- 1996e. The South American weevil genus Rhyephenes (Coleoptera: Curculionidae; Cryptorhynchinae). J. New York Entomol. Soc. 104(1-2): 1-20.

1999. Presentación preliminar de un nuevo esquema biogeográfico de América del Sur. Biogeographica 75(1): 1 16.

Morrone, J.J.\& R. S. Anderson. 1995. The Falklandius generic group: Cladistic analysis with description of new taxa (Coleoptera: Curculionidae: Rhytirrhinini). Am. Mus, Novit. 3121: 1-14.

Morrone, J.J. \& J. V. Crisci. 1995. Historical biogeography: Introduction to methods. Annu. Rev. Ecol. Syst. 26: 373-401.

Morrone, J. J., L. Katinas \& J. V. Crisci. 1997. A cladistic biogeographic analysis of Central Chile. J. Comp. Biol. 2(1): 25-41.

Morrone, J. J. \& E. C. Lopretto. 1994. Distributional patterns of freshwater Decapoda (Crustacea: Malacostraca) in southern South America: A panbiogeographic approach. J. Biogeogr. 21: $97-109$.

Morrone, J. J. \& P. Posadas. 1998. Curculionoidea. In: Morrone, J. J. \& S. Coscarón (eds.), Biodiversidad de artrópodos argentinos: Un enfoque biotaxonómico, Ediciones Sur, La Plata, pp. 258278.

Morrone, J. J. \& S. Roigm-Junent. 1995. The diversity of Patagonian weevils: An illustrated checklist of the Patagonian Curculionoidea (Insecta: Coleoptera). L.O.L.A., Buenos Aires, 189 pp. 
Morrone, J. J., S. Roig-Juñent \& J. V. Crisci. 1994. Cladistic biogeography of terrestrial Subantarctic beetles (Insecta: Coleoptera) from southern South America. Natl. Geog. Res. Expl. 10(1): 104-115.

Muiller, P. 1973. The dispersal centres of terrestrial vertebrates in the Neotropical realm: A study in the evolution of the Neotropical biota and its native landscapes. Junk, The Hague.

Muñoz, A. 1973. Descripción de Araucanobunus juberthiei gen. et sp. nov. de Triaenobini de Chile (Arachnida, Opiliones, Triaenonychidae). Physis (Buenos Aires) C, 32(84); 173-179.

Murillo, M. T. 1988. Pteridophyta- I. In: Pinto, P. \& G. Lozano (eds.), Flora de Colombia, monogr. no. 9 , Universidad Nacional de Colombia, Santafé de Bogotá, pp. 1-54,

Muzón, J. 1995. Los Odonata de la Patagonia argentina. Rev. Soc. Entomol. Argent. 54(1-4): 1-14.

Muzón, J. \& A. O. Bachmann. 1998. Plecoptera. In: Morrone, J. J. \& S. Coscarón (eds.), Biodiversidad de artrópodos argentinos: Un enfoque biotaxonómico, Ediciones Sur, La Plata, pp. 26-31.

Navas, J. R. 1977. Aves. Anseriformes. In: Ringuelet, A. A. (dir.), Fauna de Agua Dulce de la República Argentina, Fecic, Buenos Aires, 43(2): 1-94.

1991. Aves. Gruiformes. In: Castellanos, Z. A. de (dir.), Fauna de Agua Dulce de la República Argentina, Profadu (Conicet), Buenos Aires, 43(3): 179.

Nielsen, E. S. \& G. S. Robinson. 1993. Ghost moths of southern South America (Lepidoptera: Hepialidae). Entomonograph 4: 1-192.

Nowak, R. M. 1991. Walker's mammals of the World. Fifth edition. Volumes $I$ and $I I$. The John Hopkins University Press, Baltimore \& London, 1629 pp.

O'Brien, C. W. 1971. The biogeography of Chile through entomofaunal regions. Entomol. News 82: 197-207.

Olrog, C. C. 1984. Las aves argentinas: Una nuevaguia de campo. Administración de Parques Nacionales, Colección Guías de Campo no. 1, Buenos Aires, 349 pp.

Orfila, E. N. 1978. Misodendraceae de la Argentina y Chile. Fundación Elías y Ethel Malamud, Serie Científica, Buenos Aires.

Oswald, J. D. 1993. Revision and cladistic analysis of the World genera of the family Hemerobiidae (Insecta: Neuroptera). J. New York Entomol. Soc. 101(2): 143-299.

Paggi, J. C. 1998. "Cladocera" (Anomopoda y Ctenopoda). In: Morrone, J. J. \& S. Coscarón (eds.), Biodiversidad de artrópodos argentinos: Un enfoque biotaxonómico, Ediciones Sur, La Plata, pp. $507-518$.

Pakaluk, J. \& S. A. Slipinski. 1990. Review of Eupsilobinae (Coleoptera: Endemychidae) with descriptions of new genera and species from South America. Rev. Suisse Zool. 97(3): 705-728.

Peña, L. E. 1966. A preliminary attempt to divide Chile into entomofaunal regions, based on the Tenebrionidae (Coleoptera). Postilla 97: 1-17.

- 1991. El género Adelium Kirby en América (Coleoptera-Tenebrionidae). Rev. Soc. Entomol. Argent. 49(1-4): 79-84.

Peña, M. R. de la. 1992. Guía de las aves argentinas:
Tomo I. L.O.L.A., Buenos Aires.

Pereira, L. A. 1998. Chilopoda. In: Morrone, J. J. \& S. Coscarón (eds.), Biodiversidad de artrópodos argentinos: Un enfoque biotaxonómico, Ediciones Sur, La Plata, pp. 463-474.

Pereira, L. A., D. Foddai \& A. Minelli. 1997. Zoogeographical aspects of Neotropical Geophilomorpha (Chilopoda). Ent. Scand. 51: 7786.

Pereira, L. A. \& A. Minelli. 1992. A new record of Schendyloides alacer (pocock, 1891) from the Falkland islands and a redescription of species. Boll. Soc. Entomol. Ital., Genova 124(2): 83-90.

Platnick, N. I. 1983. A review of the chilensis group of the spider genus Echemoides (Araneae, Gnaphosidae). Am. Mus. Novit. 2760: 1-18.

1993. A review of the pirate spiders (Araneae, Mimetidae) of Chile. Am. Mus. Novit. 3074: 1-30.

Platrick, N. I. \& R. R. Forster. 1987. On the first American spiders of the subfamily Sternodinae (Araneae, Malkaridae).Am. Mus. Novit. 2894: 1-12.

Platnick, N. I. \& M. U. Shadab. 1983. A revision of the Neotropical spider genus Apodrassodes (Araneae, Gnaphosidae). Am. Mus. Novit. 2763: 1-14.

Porter, C. C. 1993. First record of Phrudinae (Hymenoptera: Ichneumonidae) from South America with notice of a new genus and species from Chile. 4. New York Entomol. Soc. 101(1): 130-134.

Posadas, P. E., J. M. Estévez \& J. J. Morrone. 1997. Distributional patterns and endemism areas of vascular plants in the Andean subregion. Fontqueria 48: 1-10.

Prado, E. \& R. Muñoz. 1995. Thysanoptera. In: Simonetti, J. A., M. T. K. Arroyo, A. E. Spotorno \& E. Lozada (eds.), Diversidad biológica de Chile, Conicyt, Santiago de Chile, pp. 233-240.

Ragonese, A. E. 1966. Principales recursos de la flora y fauna argentina. Curso Interamericano de Parques Nacionales y Recursos Naturales Renovables, Buenos Aires, pp. 35.

Ramírez, M. J. 1997. Revisión y fillogenia de los géneros Ferreria y Acanthoceto (Araneae: Anyphaenidae, Amaurobioidinae). Iheringia, Sér. Zool. (Porto Alegre) $82: 173-203$.

Rapoport, E. H. 1968. Algunos problemas biogeográficos del nuevo mundo con especial referencia a la región Neotropical. In: Delamare Debouteville \& E. H. Rapoport (eds.), Biologie de l'Amerique Australe, 4, CNRS, Paris, pp. 55-110.

Redford, K. H. \& J. F. Eisenberg. 1992. Mammals of the Neotropics: The southern Cone. Vol. 2. The University of Chicago Press, Chicago \& London, $430 \mathrm{pp}$.

Reichardt, H. 1977. A synopsis of the genera of Neotropical Carabidae (Insecta: Coleoptera). Quaest. Entomol. 13: 346-393.

Ringuelet, R. A. 1955a. Ubicación zoogeográfica de las Islas Malvinas. Rev. Mus. La Plata (N. S.), Zool. 6 : 419-464.

$1955 \mathrm{~b}$. Vinculaciones faunísticas de la zona boscosa del Nahuel Huapi y el dominio zoogeográfico Australcordillerano. Notas Mus. La Plata 18(160): 21-121. 
- 1961. Rasgos fundamentales de la zoogeografía de la Argentina. Physis (Buenos Aires) 22: 151-170. 1968. Llave o clave para el reconocimiento de las sanguijuelas conocidas de la República Argentina (Hirudinea) y apuntamientos sobre la hirudofauna neotrópica y transicional mexicana. Physis (Buenos Aires) $27(75)$ : $367-390$.

1975. Zoogeografía y ecología de los peces de aguas continentales de la Argentina y consideraciones sobre las áreas ictiológicas de América del Sur. Ecosur 2(3): 1-122.

1985. Annulata. Hirudinea. In: Castellanos, Z. A. de (ed.), Fauna de Agua Dulce de la República Argentina, Fecic, Buenos Aires, 17(1): 1-321.

Rivas-Martínez, S. \& G. Navarro. 1994. Mapa biogeográfico de Suramérica. Madrid.

Rivas-Martínez, S. \& O. Tovar, 1983. Síntesis biogeográfica de los Andes. Collect. Bot. (Barcelona) 14 : $515-521$

Robinson, G. S. 1984. Insects of the Falkland islands. British Museum (Natural History), London, pp. 38.

Rodriguez, R., O. Mattei \& M. Quezada. 1983. Flora arbórea de Chile. Editorial de la Universidad de Concepción, Concepción.

Roig, F. A. 1998. La vegetación de la Patagonia. In: Correa, M. N. (ed.), Flora Patagónica, tomo VIII(1), INTA, Coelcción Cientúfica, pp. 48-166.

Roig-Alsina, A. 1985. Contribución al conocimiento de los Pepsinae sudamericanos. El género Chirodamus Haliday (Hymenoptera, Pompilidae). Physis (Buenos Aires) C, 42(103): 109-120.

- 1987. Contribución al conocimiento de los Pepsinae sudamericanos. IV. El género Sphictostethus Kohl (Hymen. Pompilidae). Rev. Soc. Entomol. Argent. 44(3-4): 277-315.

Roig-Juñent, S. 1990. Revisión sistemática y análisis cladístico y biogeográfico de los géneros Cnemalobus Guérin, 1839, Cascellius Curtis, 1839 y Creobius Guérin, 1838 (Coleoptera: Carabidae). Doctoral thesis, Facultad de Ciencias Naturales y Museo, Universidad Nacional de La Plata, 234 pp.

1992a. Revisión del género Barypus Dejean, 1828 (Coleoptera, Carabidae, Broscini). Parte II. Rev. Soc. Entomol. Argent. 50(1-4): 89-117.

- 1992b. Revisión del género Barypus Dejean, 1828 (Coleoptera: Carabidae). Parte III. Rev. Soc. Entomol. Argent. 51(1-4): 1-27.

- 1994a. Las especies chilenas de Cnemalobus Guérin-Ménéille 1838 (Coleoptera: Carabidae: Cnemalobini). Rew. Chil. Entomol. 21: 5-30.

- 1994b. Historia biogeográfica de América del Sur austral. Multequina (Mendoza) 3: 167-203.

- 1995a. Cladistic analysis of Barypus Dejean 1828 (Coleoptera: Carabidae: Broscini).Am. Mus. Novit. 3117: 1-11.

1995b. Revisión sistemática de los Creobina de América del Sur (Coleoptera: Carabidae: Broscini). Acta Entomol. Chil. 19:51-74.

1998. Carabidae. In: Morrone, J. J. \& S. Coscarón (eds.), Biodiversidad de artrópodos argentinos: Un enfoque biotaxonómico, Ediciones Sur, La Plata, pp. $194-209$.

Roig-Junent, S. \& G. E. Ball. 1995. Nothobroscus chilensis, new genus and new species, from southern South America (Coleoptera: Carabidae: Broscini). Coleopts. Bull. 49(4): 301-312.

Roig-Juñent, S. \& A. C. Cicchino. 1989. Revisión del género Barypus Dejean, 1828 (Coleoptera: Carabidae: Broscini). Parte 1. Bol. Soc. Biol. Concepción 60: 201-225.

Ronderos, R. A. \& S. Turk. 1989. Notas sobre Melanoplinae neotropicales (Orthoptera, Acrididae). Rev. Soc. Entomol. Argent. 46(1-4): 69-74.

Rothkügel, M. 1916. Los bosques patagónicos. Ministerio de Agricultura, Oficina de Bosques y Yerbales, Buenos Aires, pp. 204.

Rühm, W. 1987. Zur Biologie, Ökologie und Phänologie des Calvertius tuberosus Ger. \& Ferm. (Col., Curculionidae) auf des Araucarie (Araucaria araucana (Mol.) Koch). Entomol. Mitteil.Zool. Mus. Hamburg 9(130): 1-7.

Sanders, R. W., T. F. Stuessy, C. Marticorena \& M. Silva. 1987. Phytogeography and evolution of Dendroseris and Robinsonia, tree-Compositae of the Juan Fernandez islands. Op. Bot. 92: 195-215.

Schiapelli, R. D. \& B. S. Gershman de Pikelin. 1968. El género Scotinoecus Simon, 1892 (Araneae, Dipluridae, Hexathelinae). Physis (Buenos Aires) 27(75): $313-322$.

Schmid, F. 1989. Les hydrobiosides (T'richoptera: Annulipalpia). Bull. Inst. Roy. Sci. Nat. Belgique 59: $1-154$.

Schoorl, J. W. Jr. 1989. A phylogenetic study on Cossidae (Lepidoptera: Ditrysia) based on external adult morphology. Zool. Verh. Leiden 263: 1-138.

Seberg, O. 1991. Biogeographic congruence in the south Pacific. Aust. Syst. Bot. 4: 127-136.

Shannon, R. C. 1927. Contribución a los estudios de las zonas biológicas de la República Argentina. Rev. Soc. Entomol. Argent. 4: 1m14.

Sibley, C. G. \& B. L. Monroe. 1990. Distribution and taxonomy of birds of the world. Yale University Press, New Haven \& London, $1111 \mathrm{pp}$.

Sick, W. D. 1969. Geographical substance. Mon. Biol. 19: $449-474$.

Skottsberg, C. 1921. The vegetation in South Georgia. In: Nordenskjöld, O. (ed.), Wissenschaftliche Ergebnisse der Schwedischen Sudpolar Expedition 1901-1903, 4 Botanik, (12), pp. 1-36.

Solervicens, A. J. 1986. Revisión taxonómica del género Eurymetopum Blanchard, 1844 (Coleoptera, Cleridae, Phyllobaeninae). Acta Entomol. Chil. 13: $11-120$.

- 1987. Filogenia y biogeografía del género Eurymetopum Blanchard, 1844 (Coleoptera: Cleridae: Phyllobaeninae). Acta Entomol. Chil. 14: 127-154.

Solomon, J. C. 1982. The systematics and evolution of Epilobium (Onagraceae) in South America. Ann. Missouri Bot. Gard. 69: 239-335.

Soriano, A. 1950. La vegetación del Chubut. Rev. Argent. Agric. 17(1): 30-66.

Stock, J. H. \& D. Platvoet. 1991. The freshwater Amphipoda of the Falkland islands. J. Nat. Hist. 25: 1469-1491.

Straneo, S. L. 1951. Nuovi Pterostichini VII (Coleoptera: Carabidae). Doriana 1(36): 1-12. 
Straneo, S. L. \& R. Jeannel. 1955. Los insectos de las islas Juan Fernández. 23. Carabidae (Coleoptera). Rev. Chil. Entomol. 4: 121-144.

Stuessy, T. F., D. J. Crawford \& C. Marticorena. 1990. Patterns of phylogeny in the endemic vascular flore of the Juan Fermandez islands, Chile. Syst. Bot. 15(2): 338-346.

Stuessy, T. F., R. W. Sanders \& M. Silva. 1984. Phytogeography and evolution of the flora of the Juan Fernandez islands: A progress report. In: Radowsky, F. J., P. H. Raven \& S. H. Sohmer (eds.), Biogeography of the tropical Pacific: Proceedings of a symposium, Association of Systematics Collections and Bernice P. Bishop Museum, Kansas, pp. 55-69.

Takhtajan, A. Floristic regions of the world. University of California Press, Berkeley.

Thayer, M. K. 1989. Biology and phylogenetic relationships of Neophonus bruchi, an anomalous south Andean staphylinid (Coleoptera). Syst. Entomol. 12: 389-404.

Troncoso, A. \& E. J. Romero. 1998. Evolución de las comunidades florísticas en el extremo sur de Sudamérica durante el Cenofítico. In: Proceedings of the VI Congreso Latinoamericano de Botánica (1994), Monographs in Systematic Botany from the Missouri Botanical Garden 68, pp. 149-172.

Tuhkanen, S., I. Kuokka, J. Hyvönen, S. Stenroos \& J. Niemelä. 1990. Tierra del Fuego as a target for biogeographical research in the past and present. An. Inst. Patagonia (Punta Arenas) 19(2): 1-107.

Vanin, S. A. 1976. Taxonomic revision of the South American Belidae (Coleoptera). Arq. Zool. Sâo Paulo 28(1): $1-75$.

Vitali-Di Castri, V. 1970. Un nuevo género de Gymnobisinae (Pseudoscorpionida) de las islas Malvinas. Revisión taxonómica de la subfamilia. Physis (Buenos Aires) 30(80): 1-9.

- 1974. Presencia en América del Sur del género Sathrochthonius (Pseudoscorpionida) con descripción de una nueva especie. Physis (Buenos Aires) 33(87): 193-201.

- 1975. Nuevos Austrochthonius sudamericanos (Pseudoscorpionida, Chthonidae). Physis (Buenos Aires) C, $34(89): 117-127$.

Voisin, J.-F. 1987. Sur les coléoptères des îles Falkłand, notes et signalisations. Bull. Soc. Entomol. France 91(3-4): $93-95$.

Weston, P. H. \& M. D. Crisp. 1987. Evolution and biogeography of the waratahs. In: Armastrong, J. A. (ed.), Waratahs: Their biology, cultivation and conservation, Australian National Botanic Gardens Occasional Publication 9, pp. 17-34.

1994. Cladistic biogeography of waratahs (Proteaceae: Embothriae) and their allies across the Pacific. Aust. Syst. Bot. 7: 225-249.

- 1996. Trans-Pacific biogeographic patterns in the Proteaceae. In: Keast, A. and S. E. Miller (eds.), The origin and evolution of Pacific islands biotas, New Guinea to eastern Polynesia: Patterns and processes, Academic Publishing, Amsterdam, pp. $215-232$.

Wibmer G. J. \& C. W. O'Brien. 1986. Annotated checklist of the weevils (Curculionidae sensu lato) of South America (Coleoptera: Curculionoidea). Mem. Am. Entomol. Inst. 39: 1-563.

Williams, J. D. \& D. D. Echeverría. 1995. Amphibia. In: Lopretto, E. C. \& G. Tell (eds.), Ecosistemas de aguas continentales: Metodologías para su estudio, III, Ediciones Sur, La Plata, pp. 1369-1401.

Wood, S. L. 1986. A reclassification of the genera of Scolytidae (Coleoptera). Great Basin Nat. Mem. 10: 1-126.

Wygodzinsky, P. 1967. On the geographical distribution of the South American Microcoryphia and Thysanura (Insecta). In: Delamare Debouteville \& E. H. Rapoport (eds.), Biologie de l'Amerique Australe, 3, CNRS, Paris, pp. 505-524.

Wygodzinsky, P. \& S. Coscarón. 1989. Revision of the black fly genus Gigantodax (Diptera: Simulidae). Bull. Am. Mus. Nat. Hist. 189: 1-269.

Zavaro, C. A., J. V. Crisci \& J. J. Morrone. 1997. Synopsis and cladistics of the genus Misodendrum (Misodendraceae, Santalales). Fontqueria 48: 225239.

Zunino, M. 1984. Analisi sistematica e zoogeografica della sottofamiglia Taurocerastinae Germain (Coleoptera, Scarabeoidea: Geotrupidae). Boll. Mus. Reg. Sci. Nat. Torino 2(2): 445-464.

Recibido: 13-XII-1999 Aceptado: 27-III-2000 